\title{
Experimental Setup for the Investigation of Reproducibility of Novel Tool Changing Systems in Nanofabrication Machines
}

\author{
Florian Weigert $^{1}\left[\right.$ Roman Hebenstreit $^{1} \cdot$ Roland FüßI $^{2} \cdot$ René Theska $^{1}$
}

Received: 20 December 2020 / Revised: 2 March 2021 / Accepted: 4 March 2021 / Published online: 5 May 2021

(c) The Author(s) 2021

\begin{abstract}
Nanomeasuring machines developed at the Technische Universität Ilmenau enable three-dimensional measurements and manufacturing processes with the lowest uncertainties. Due to the requirements for these processes, a highly reproducible and long-term stable tool changing system is needed. For this purpose, kinematically determined couplings are widely used. The state-of-the-art investigations on those are not sufficient for the highest demands on the reproducibility required for this application. A theoretical determination of the reproducibility based on analytical or numerical methods is possible, however not in the desired nanometer range. Due to this, a measurement setup for the determination of the reproducibility in five degrees of freedom with nanometer uncertainty was developed. First, potential measuring devices are systematically examined and measurement principles were developed out of this. A three-dimensional vector-based uncertainty analysis is performed to prove the feasibility of the measurement principle and provides a basis for further design. As a result, a translatory measurement uncertainty of $10 \mathrm{~nm}$ and a rotatory uncertainty of $11 \mathrm{nrad}$ can be reached. Afterwards, the measurement setup is designed, focusing on the metrological frame and the lift-off device. The developed setup exceeds the uncertainties of the measurement setups presented in the state-of-the-art by an order of magnitude, allowing new in-depth investigations of the reproducibility of kinematic couplings.
\end{abstract}

Keywords Tool changing $\cdot$ Kinematic coupling $\cdot$ Measurement setup $\cdot$ Uncertainty analysis $\cdot$ Precision design

\section{Introduction}

The further development in the field of nanofabrication constantly creates new challenges for the machines. At the Technische Universität Ilmenau, the existing nanomeasuring machines are currently being extended to allow

\author{
Florian Weigert \\ florian.weigert@tu-ilmenau.de \\ Roman Hebenstreit \\ roman.hebenstreit@tu-ilmenau.de \\ Roland Füß1 \\ roland.fuess1@tu-ilmenau.de \\ René Theska \\ rene.theska@tu-ilmenau.de
}

1 Institute for Design and Precision Engineering, Precision Engineering Group, Technische Universität Ilmenau, Gustav-Kirchhoff-Platz. 2, 98693 Ilmenau, Germany

2 Institute for Process Measurement and Sensor Technology, Technische Universität Ilmenau, Gustav-Kirchhoff-Str. 1, 98693 Ilmenau, Germany nanofabrication [1, 2]. A highly reproducible tool-changing system is required for combined processes with multiple sensors and tools. Previous solutions for this are based on a calibration process using the measurement of markers, which increases the processing time considerably and manufacturing tools may not have their own measuring system. The targeted three-dimensional reproducibility of the position of the tool-changing system is related to the three-dimensional measurement uncertainty of the nanomeasuring machine NPMM-200 and lies below $50 \mathrm{~nm} \mathrm{[3].}$

The tool-changing interface is based on kinematic coupling, which is characterized by six ball-plane contacts and is therefore free of overdetermination. In the literature, the types shown in Fig. 1 are known. The Maxwell arrangement consists of three $\mathrm{V}$-grooves and is characterized by a central pivot point based on its symmetrical design. The Kelvin arrangement has a fixed pivot point realized by the ball-tetrahedron pairing. One of the major influencing factors on the reproducibility of kinematic couplings is the frictional behavior of the ball-plane contacts. For analytical or numerical calculations of the reproducibility, idealized 


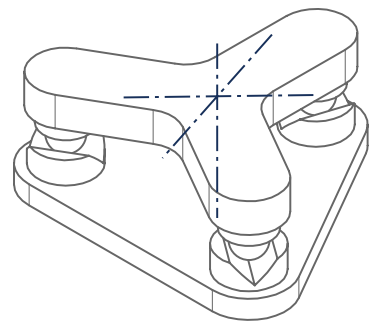

(a)

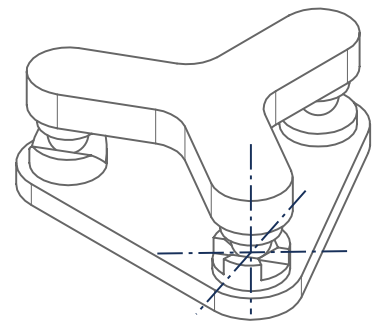

(a)
Fig. 1 Kinematic couplings: a Maxwell clamp [4], b Kelvin clamp [5]

considerations of position and geometry of the contact surfaces are necessary. The friction occurring at the mechanical contact points during the coupling process can only be considered simplified. These simplifications are no longer acceptable for the demands made on reproducibility in the research activity presented here. Therefore, a measurement setup is required for the experimental determination of the reproducibility.

\section{State of the Art}

State-of-the-art kinematic couplings are widely used in precision engineering and have already been investigated for their achievable reproducibility in various measurement setups. In coordinate measuring machines, kinematic couplings are used as stylus-changing interfaces. The performance is not specified, however, a recalibration after a stylus change is not necessary. Therefore, the reproducibility must be clearly below the measurement uncertainty of the machine. For the most accurate coordinate measuring machines available on the market, the accuracy is specified with $0.3+L / 1000 \mu \mathrm{m}$ [6].

In the 1980 s, the reproducibility of kinematic couplings was already extensively investigated by the MIT precision engineering research group of Slocum [7, 8]. The kinematic couplings in the Maxwell arrangement are intended for use in tooling machines. This results in a coupling diameter of $356 \mathrm{~mm}$ and a ball diameter of $28.6 \mu \mathrm{m}$. The preload force of $45 \mathrm{kN}$ was provided by a pneumatic piston, which is also used for the automated lift-off process. Lubricated and nonlubricated sets of coupling elements are investigated for 600 cycles. The measurement of the reproducibility is carried out using a total of six linear variable differential transformers (LVDT), arranged one each in the radial and axial direction at three points at an angle of $120^{\circ}$ to each other. Thus, the measurement in all six degrees of freedom (DOF) is possible. Temperature stabilization of $\pm 1 \mathrm{~K}$ was reached, although the influence of temperature effects is minimal due to the massive design.
In their research, Grimske et al. focuses on the investigation of kinematic couplings with a desired reproducibility of $1 \mu \mathrm{m}$ for small tooling machines [9]. For the determination of the reproducibility, five fiber-coupled semiconductor interferometers with a resolution of $0.025 \mathrm{~nm}$ are used, which are mounted on a monolithic aluminum frame. The preload force of the coupling is applied by magnets and the measurement is performed for 25 cycles with a manual liftoff device.

In [10], a kinematic mirror mount for ultra-precision manufacturing with a corresponding measuring setup is presented. This mirror mount is based on a rotatory coupled kinematic coupling with a diameter of the moving coupling half of $270 \mathrm{~mm}$. The measurement is performed in five DOF using five capacitive probes with a resolution of 10 nm mounted on a multi-piece metrological frame made of aluminum. The lift-off process is done manually, the measurement was performed under atmospheric conditions with temperature stabilization of $\pm 0.1 \mathrm{~K}$. Multiple sets of 30 cycles are performed after a break-in process and subsequent cleaning and lubricating of the coupling elements.

The work of Ziegert and Tymianski deals with the development of an air-bearing kinematic coupling [11]. The reproducibility of those air-bearing couplings is measured with six capacitive sensors in six DOF. The sensors are attached by additional parts to the metrological frame, which is made of aluminum like the moving half of the coupling. The resolution of the capacitive sensors was determined experimentally throughout $24 \mathrm{~h}$ and equals $18 \mathrm{~nm}$. Information about temperature stabilization is not given. The process is measured for 1000 cycles using a pneumatic cylinder for an automated lift-off process.

Other approaches are based on optical tracking methods $[12,13]$. Either a marker or an illuminated pinhole is used, whose position is detected by an imaging system. This only allows measurement up to three DOF. In [12], a standard deviation of the reproducibility of $30 \mathrm{~nm}$ is given. According to [13], a resolution in the subnanometer range is possible, but only under certain conditions. The number of cycles for a pair of coupling elements is not specified in both works.

\section{Objectives for the Development of the Measurement Setup}

The investigations on kinematic couplings presented in the state of the art were carried out under conditions and objectives that considerably vary from the intended application in the nanofabrication machine. For this reason, the measurement setups are not capable of an investigation of the reproducibility with the precision required in this research. Furthermore, up to now, no measuring setup has been designed for operation under vacuum conditions. 
The aim of this work is the development of a measurement setup for the determination of the reproducibility of the position of a kinematic coupling in a vacuum. The measurement uncertainty is intended to be at least five times lower then the desired three-dimensional reproducibility of $50 \mathrm{~nm}$. Since a rotation around the tool axis is not relevant for the intended application, the measurement must be carried out in five DOF. The kinematic coupling has to be integrated into the measurement setup in a manner that allows the coupling elements to be exchanged to investigate different shapes and materials.

The development is carried out systematically and starts with a detailed analysis of measuring devices. Based on this, measuring principles are developed, evaluated, and selected. The measurement uncertainty is determined through a measurement uncertainty analysis according to the Guide to the Expression of Uncertainty in Measurement (GUM). Subsequently, the measuring setup is designed.

\section{Synthesis of a Measurement Principle}

For the investigation of the reproducibility, the two halves of the kinematic coupling have to be separated, coupled again, and preloaded with a defined force. The position of the moved coupling half relative to the stationary coupling half is measured in five DOF. Therefore, the sensors are attached to the fixed coupling half and the measuring surfaces are located on the moved coupling half. To exclude influences on the reproducibility by a change of the preload force, the force resulting from the mass of the moved coupling half is used as preload force. The measurement procedure needs to be performed a sufficient number of cycles using an automated lift-off device.

For this purpose, measuring principles are developed based on the potential measurement devices shown in Tables 1 and 2. These measurement devices are preselected concerning the requirements of the measuring setup. For the development of the measurement principles, these measurement devices are combined to measure three translatory and two rotatory DOF independently. The aim is to use one type of measuring device only to reach a simple measuring setup and to reduce the number of parasitic effects. Small angular deviations can be captured via triangulation by differential length measurement as well. A total of 13 measuring principles are developed and evaluated based on the following evaluation criteria.

- Short curcuited metrological loop

- Vacuum compatibility

- Setup reduced to the most necessary, open extensions

- Ease of integration of an automated lift-off for automated measurements

The solution based on five single-electrode capacitive sensors showed the best performance. These sensors are very compact, vacuum compatible, and can be combined easily for measuring five DOF independently. The measuring arrangement is shown in Fig. 2. Two sensors are used to measure the $x$ and $y$ reproducibility. Three further sensors are used to measure the reproducibility in the $z$-direction and the rotatory reproducibility around the $x$ - and $y$-axis.

Table 1 Typical properties of high-precision translatory measuring devices achieved in the state of the art [13-16]

\begin{tabular}{lllllc}
\hline $\begin{array}{l}\text { Measurement } \\
\text { principle }\end{array}$ & Measuring device & Resolution $(\mathrm{nm})$ & $\begin{array}{l}\text { Measuring range } \\
(\mathrm{mm})\end{array}$ & Contactless & Relative/absolute \\
\hline Capacitive & Capacitive sensors & $0.1-1$ & $0.02-10$ & Yes & Absolute \\
Inductive & $\begin{array}{l}\text { Linear variable differential transformer } \\
\text { (LVDT) }\end{array}$ & $10-100$ & $1-600$ & No & Absolute \\
Tracking & Lighted-pinhole-tracking & 1 & $0.01 \times 0.01$ & Yes & Absolute \\
Confocal & Focus sensor & $0.1-10$ & $0.002-1$ & Yes & Absolute \\
Confocal & Chromatic confocal & 10 & $0.3-30$ & Yes & Absolute \\
Optical & Laser interferometer & 0.01 & 100 & Yes & Relative \\
Optical & Position sensitive detector (PSD) & $10-100$ & $2 \times 2$ & Relative &
\end{tabular}

Table 2 Typical properties of high-precision rotatory measuring devices achieved in the state of the art $[17,18]$

\begin{tabular}{llllll}
\hline Measuring device & $\begin{array}{l}\text { Resolution } \\
(\mu \mathrm{rad})\end{array}$ & $\begin{array}{l}\text { Measuring } \\
\text { range }(\mathrm{mrad})\end{array}$ & $\begin{array}{l}\text { Number } \\
\text { of DOF }\end{array}$ & Contactless & Relative/absolute \\
\hline Autocollimator & 0.5 & 8.7 & 2 & Yes & Absolute \\
Inclinometer & 0.6 & 9 & 1 & No & Absolute \\
Three-beam interferometer & 0.005 & 0.1 & 2 & Yes & Absolute \\
\hline
\end{tabular}




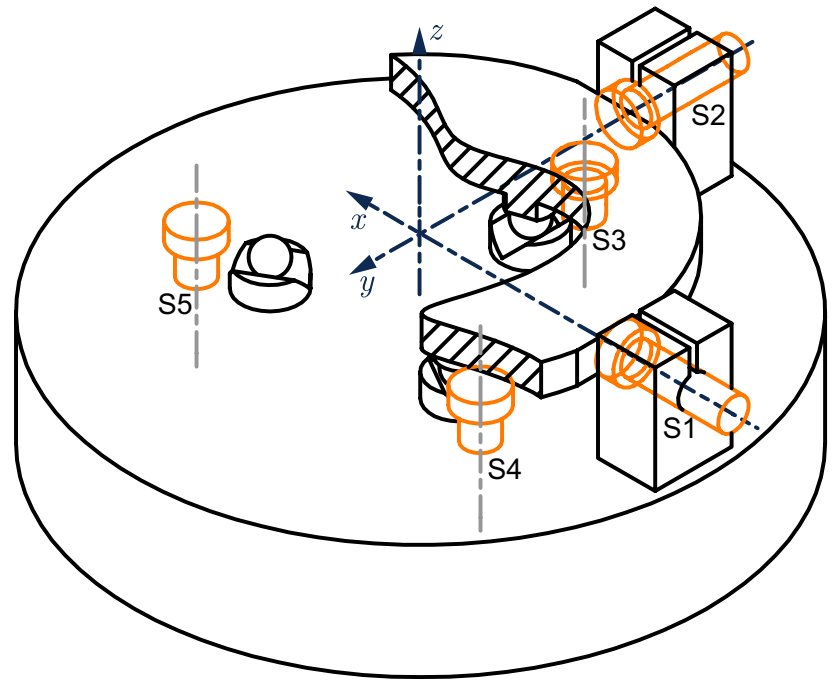

Fig. 2 Measurement arrangement

The selected sensors have a static resolution of $0.5 \mathrm{~nm}$ and a measuring range of $50 \mu \mathrm{m}$. For the following uncertainty analysis materials, dimensions, sensor positions, and environmental conditions are specified.

\section{Uncertainty Analysis According to the GUM}

To prove the capability of the selected measurement arrangement and for the design of the measurement setup, an uncertainty analysis must be performed. This uncertainty analysis is based on the Guide to the Expression of Uncertainty in Measurement (GUM). A vector-based approach, which has already been proven to be successful for modeling the uncertainty of the NPMM-200, is chosen [3]. Individual uncertainty influences are modeled as subvectors and combined to build a closed vector chain. Advantageous is the good expandability and modifiability of this chain.

\subsection{Uncertainty Models for the Sensors}

The modeling is based on the analogy between the measurement setup and a positioning system with six DOF. The range of the moving coupling half corresponds to the worst expected reproducibility of the kinematic coupling. The vector chains are created for the two states $a$ and $b$. In state $a$, the moving coupling half is located at the negative maximum and in state $b$ at the positive maximum of the reproducibility. For the calculation of the overall uncertainty, the uncertainty of the difference vector $\boldsymbol{r}_{\text {meas }}$ between the two states $a$, and $b$ is used.

The vector chain for the uncertainty in $x$ - and $y$-direction is identical and shown in Fig. 3. It consists of the

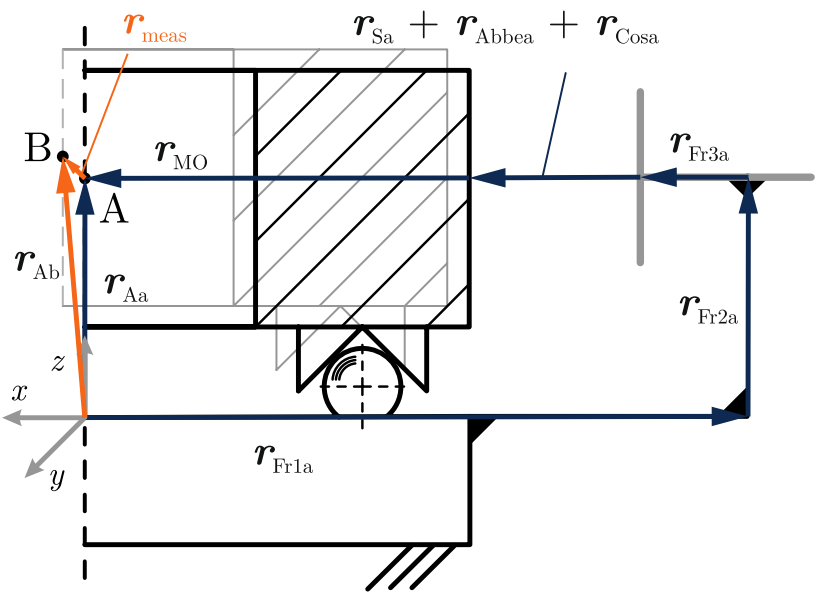

Fig. 3 Vector chain for the uncertainty calculation in $x$ and $y$ direction for state $a$ (state $b$ shown schematically in gray)

frame vectors $\boldsymbol{r}_{\mathbf{F r}}$ and measurement object vectors $\boldsymbol{r}_{\mathbf{M O}}$ as well as the measurement value of the sensor $\boldsymbol{r}_{\mathbf{S}}$. First-order Abbe error (sine error) $\boldsymbol{r}_{\text {Abbe }}$ and second-order Abbe error (cosine error) $\boldsymbol{r}_{\text {Cos }}$ are expected hereby. For both states $a$ and $b$, the equation for the vector chain results in:

$0=\boldsymbol{r}_{\mathrm{A}_{\mathrm{a}}}-\boldsymbol{r}_{\mathrm{MO}_{\mathrm{a}}}-\boldsymbol{r}_{\mathrm{S}_{\mathrm{a}}}-\boldsymbol{r}_{\mathrm{Abbe}_{\mathrm{a}}}-\boldsymbol{r}_{\mathrm{Cos}_{\mathrm{a}}}-\sum_{i=1}^{4} \boldsymbol{r}_{\mathrm{Fr}_{\mathrm{ia}}}$

$0=r_{\mathrm{A}_{\mathrm{b}}}-\boldsymbol{r}_{\mathrm{MO}_{\mathrm{b}}}-\boldsymbol{r}_{\mathrm{S}_{\mathrm{b}}}-\boldsymbol{r}_{\mathrm{Abbe}_{\mathrm{b}}}-\boldsymbol{r}_{\mathrm{Cos}_{\mathrm{b}}}-\sum_{i=1}^{4} \boldsymbol{r}_{\mathrm{Fr}_{\mathrm{ib}}}$

$r_{\text {meas }}=r_{\mathrm{A}_{\mathrm{a}}}-r_{\mathrm{A}_{\mathrm{a}}}$

The modeling of the $z$-direction differs slightly from the $x$ - and $y$-direction and is shown in Fig. 4. Again, the difference vector is formed for two states, but for a better overview, this is not shown in the figure. The vector chain consists of the frame vectors $\boldsymbol{r}_{\mathrm{Fr}}$ and the measurement vectors of the sensors $\boldsymbol{r}_{\mathrm{S}}$, whereby second-order Abbe errors $r_{\text {Cos }}$ are considered. Initially, the translatory uncertainty in the $z$-direction is determined by averaging the three sensors.

$r_{z}=\frac{1}{3} \cdot \sum_{i=1}^{3} s_{i a}$

For the determination of the rotatory uncertainties, a plane defined by the vectors $\boldsymbol{p}, \boldsymbol{v}$ and $\boldsymbol{u}$ is created and the corresponding normal vector $\boldsymbol{n}$ is calculated. The angle of the 


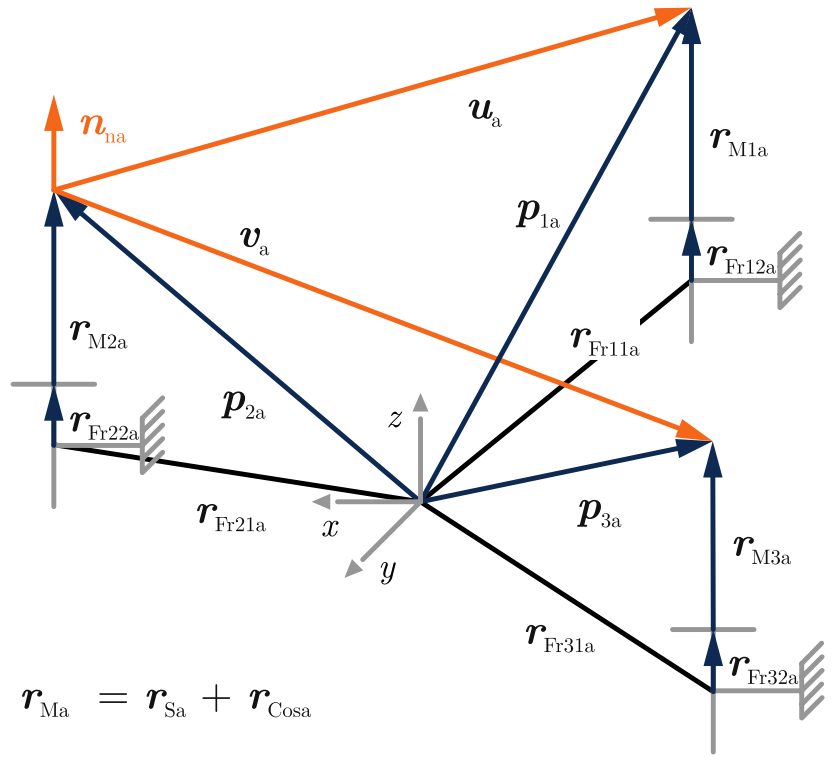

Fig. 4 Vector chain for the uncertainty calculation in $z$ direction and around the $x$ - and $y$-axis for state $a$

plane is calculated by the angle between the normal vector of the sensor plane and the respective coordinate axis:

$\epsilon_{x}=\pi / 2-\arccos \left(\frac{n_{y}}{\sqrt{n_{x}^{2}+n_{y}^{2}+n_{z}^{2}}}\right)$

$\epsilon_{y}=\pi / 2-\arccos \left(\frac{n_{x}}{\sqrt{n_{x}^{2}+n_{y}^{2}+n_{z}^{2}}}\right)$

The determination of the angles is again done for the two states $a$ and $b$. The uncertainty is calculated from the uncertainty of the difference angle $\epsilon_{\text {meas }}$.

$\epsilon_{\text {meas }}=\epsilon_{b}-\epsilon_{a}$

\subsection{Uncertainty Contributions}

In the following, the individual uncertainty contributions, each modeled as a single vector, are introduced.

\subsubsection{Frame Components}

The vectors for the frame components $\boldsymbol{r}_{\mathbf{F r}}$ are derived from the measuring arrangement. Uncertainties due to position deviations of the sensors $b_{\text {sensor }}$ are considered hereby. Besides, thermal expansions also have a significant influence on the measurement uncertainty of the entire system. These were calculated according to Eq. (8) considering the current temperature $\theta_{\text {cur }}$ and the thermal expansion coefficient $\alpha_{\text {th }}$ at a reference temperature of $20^{\circ} \mathrm{C}$.

$\boldsymbol{r}_{\mathbf{F r}}=\boldsymbol{r}_{\mathbf{F r}_{20^{\circ} \mathrm{C}}} \cdot\left(1+\alpha_{\mathrm{th}} \cdot\left(\theta_{\text {cur }}-20^{\circ} \mathrm{C}\right)\right)$

\subsubsection{First-Order Abbe Errors (Sine Error)}

Due to the rotary and translatory motion of the upper coupling half, first-order Abbe errors occur. These errors occur when the measuring axis is not aligned with the motion axis. The entire measurement setup works as a relative measurement and the motion axis is in a random position. For this reason, the worst expected translatory reproducibility $a_{\text {rep }}$ is assumed to be the distance $r_{\text {Dis }}$ between the measuring and motion axis. The tilt angle $\beta_{\text {Abbe }}$ equals the worst expected rotatory reproducibility $\alpha_{\text {rep }}$.

$r_{\text {Abbe }}=\tan \left(\beta_{\text {Abbe }}\right) \times r_{\text {Dis }}$

Due to the small angles $\beta_{A b b e}$ in the range of multiple $\mu \mathrm{rad}$ the model can be linearized according to Eq. (10).

$r_{\text {Abbe }}=\beta_{\text {Abbe }} \times r_{\text {Dis }}$

\subsubsection{Second-Order Abbe Errors (Cosine Error)}

Second-order Abbe errors occur when the sensors are tilted by the angle $\gamma$. The distance between the sensor and measuring object equals the measurement value of the sensors $r_{\mathrm{C}}$.

$\boldsymbol{r}_{C o s}=\boldsymbol{r}_{C} \circ\left(\begin{array}{l}1 \\ 1 \\ 1\end{array}\right) \times(1-\boldsymbol{\gamma})$

\subsubsection{Non-Orthogonal Coordinate System}

Due to manufacturing tolerances, the coordinate system defined by the sensor axes is not perpendicular. This uncertainty is corrected by a coordinate transformation from the non-orthogonal coordinate system $\boldsymbol{r}_{\text {not orth }}$ to the orthogonal coordinate system $\boldsymbol{r}_{\text {orth }}$ using the linear transformation matrix $A_{\text {lin }}$ according to [19]. The tilt of the sensors is identical to $\gamma$ and is described as $\Delta \varphi$ for this sub-model. For the $z$-direction the means values of the three sensors are combined to a virtual sensor, which coincides with the coordinate axis.

$\boldsymbol{r}_{\text {orth }}=A_{\text {lin }} \cdot \boldsymbol{r}_{\text {not orth }}$ 
$A_{\text {lin }}=\left[\begin{array}{ccc}1 & -\Delta \varphi_{x y} & -\Delta \varphi_{x z} \\ 0 & 1 & \Delta \varphi_{y z} \\ 0 & 0 & 1\end{array}\right]$

Afterwards, the difference of the vectors can be calculated.

$\Delta r_{\text {meas }}=\left|r_{\text {orth }}\right|-\left|r_{\text {not orth }}\right|$

\subsection{Calculated Measurement Uncertainty}

The measurement uncertainty is calculated based on the created model. For this purpose, uncertainties and uncertainty distributions are assigned to the variables introduced in the model. Since these are based on manufacturer specifications, manufacturing tolerances, or conservative estimations, a rectangular distribution (type B) is assumed for all influencing factors.

In Table 3, all influencing parameters on the measurement uncertainty are shown. The worst expected reproducibility of the position of the kinematic coupling is estimated according to the state of the art $[8,9,12,13]$. Measuring range, resolution, material of the sensors, and permissible tilt of the sensor axis relative to the measuring surface are given in the datasheet of the manufacturer [20]. The measurement uncertainty of the sensors for a static measurement is estimated as factor two of the sensor resolution. Sensor positions are derived by the use of a coordinate measuring machine. Thus, the uncertainty is given by this measurement. All components in the metrological circle of the measuring setup are made of the glass-ceramic Zerodur ${ }^{\circledR}$, which has a thermal expansion coefficient close to zero [21].

Subsequently, the measurement uncertainty can be calculated. This results in $9.00 \mathrm{~nm}$ uncertainty in the $x$-direction, $8.76 \mathrm{~nm}$ uncertainty in the $y$-direction, and $10.06 \mathrm{~nm}$ uncertainty in the $z$-direction with a coverage factor $k=1$. Figure 5 shows the uncertainty contributions for the translatory

Table 3 Assumptions of the values for the measurement uncertainty

\begin{tabular}{lll}
\hline Description & Symbol & Value \\
\hline Expected translatory reproducibility & $a_{\text {rep }}$ & $5 \mu \mathrm{m}$ \\
Expected rotatory reproducibility & $\alpha_{\text {rep }}$ & $10 \mu \mathrm{rad}$ \\
Nominal measuring range of the sen- & $d_{\text {sensor }}$ & $50 \mu \mathrm{m}$ \\
$\quad$ sors & & \\
Measurement uncertainty of the sen- & $\delta_{\text {sensor }}$ & $1 \mathrm{~nm}$ \\
$\quad$ sors & & \\
Translatory deviation of the sensor & $b_{\text {sensor }}$ & $10 \mu \mathrm{m}$ \\
$\quad$ position & & \\
Rotary deviation of the sensor axes & $\gamma, \Delta \varphi$ & $700 \mu \mathrm{rad}$ \\
Temperature & $\theta$ & $20^{\circ} \pm 0.05 \mathrm{~K}^{-1}$ \\
Thermal expansion coefficient of frame & $\alpha_{\text {frame }}$ & $0 \pm 0.1 \mathrm{~K}^{-1}$ \\
Thermal expansion coefficient of & $\alpha_{\text {sensor }}$ & $13 \cdot 10^{-6} \pm 0.1 \mathrm{~K}^{-1}$ \\
$\quad$ sensor & & \\
\hline
\end{tabular}

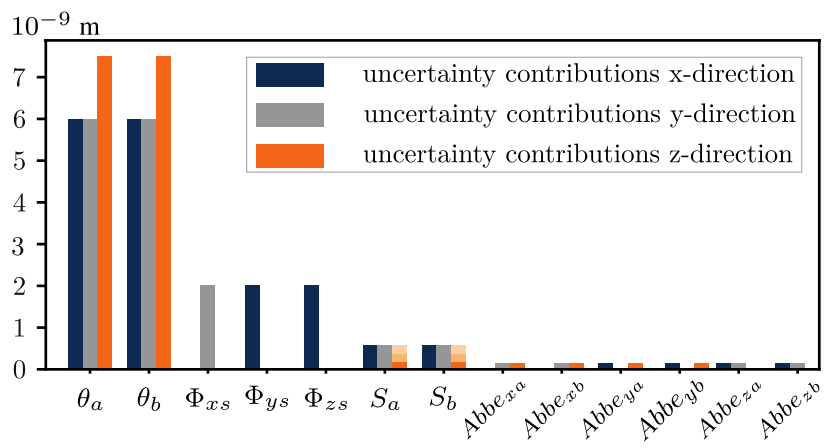

Fig. 5 Uncertainty contributions for the translatory measurement uncertainty

measurement uncertainty. It becomes clear that the largest uncertainty contributions are given by the thermal expansions of frame and sensors. Furthermore, the non-orthogonal coordinate axes and the measurement uncertainty of the sensors themselves influence the overall uncertainty. First-order Abbe errors also have a small influence. Influencing factors not shown have either no or negligible influence on the measurement uncertainty.

For the measurement of the rotatory reproducibility, relatively small measurement uncertainties of $10.7 \mathrm{nrad}$ around the $x$ - and $y$-axis was achieved $(k=1)$. These small uncertainties can be explained by the measuring principle, since the arrangement of the sensors is symmetrical. Thermal expansion has the same effects on all sensors and therefore does not contribute to the uncertainty. For this reason, the uncertainty amounts for the rotatory measurement uncertainty are given only by the measurement uncertainties of the sensors themselves and position deviations of the sensors, as shown in Fig. 6.

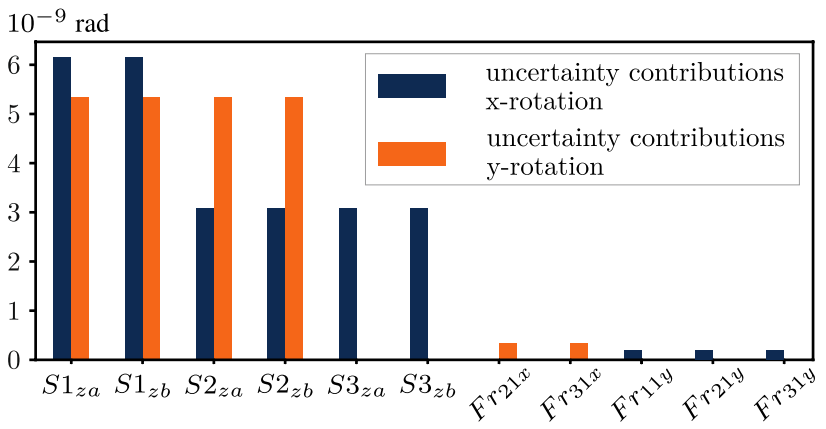

Fig. 6 Uncertainty contributions for the rotatory measurement uncertainty 


\section{Design of the Measurement Setup}

The design is based on the performed measurement uncertainty analysis and is divided into the main assemblies: Metrological frame with clamping of the sensors and a lift-off device.

\subsection{Preliminary Tests of the Clamping of the Capacitive Sensors}

The sensors need to be clamped highly reproducible and

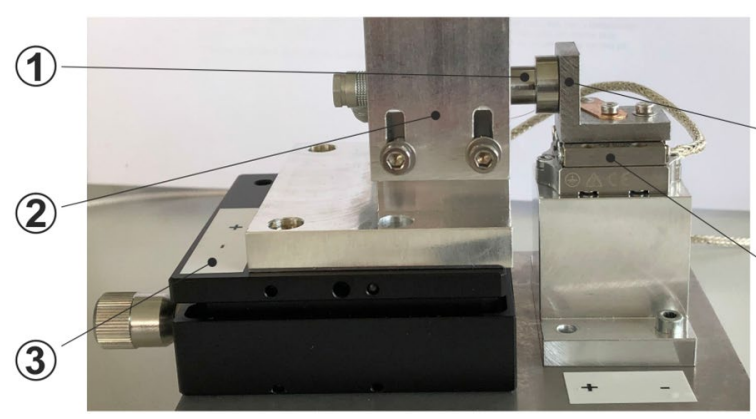

Fig. 7 1-axis test setup of the sensor clamping: (1) capacitive sensor; (2) clamping of sensor in V-groove ; (3) 2-axis rotatory adjustment table; (4) capacitor measuring plate; (5) linear axis

stable. For this purpose, the cylindrical capacitive sensors are placed into V-grooves, preloaded by a small and defined spring force. An adjustment of the sensors into the working distance of $50 \mu \mathrm{m}$ is done using gauge blocks. Thereby, the permissible angular deviation of $700 \mu \mathrm{rad}$ of the sensor is checked and, if necessary adjusted using thin spacer elements.

This variant of sensor clamping and sensor adjustment was investigated in a 1-axis pre-test which is shown in Fig. 7. The sensor is mounted on a two-axis rotatory adjustment table. The measuring surface of the sensor is placed on a linear axis with a resolution of $8 \mathrm{~nm}$. This was moved in defined steps and the position was measured by the capacitive sensor and the integrated measuring system of the axis. The closed-loop control was switched off for the duration of the measuring value acquisition to achieve static measurement. The measured values of the capacitive sensor and the integrated measuring system show deviations of $0.4 \mu \mathrm{m}$. These deviations are very reproducible and caused by movements after deactivating the closed-loop control of the axis.

\subsection{Metrological Components}

The metrological frame consists of a static and moving half, and is shown in Fig. 8. The static half is primarily

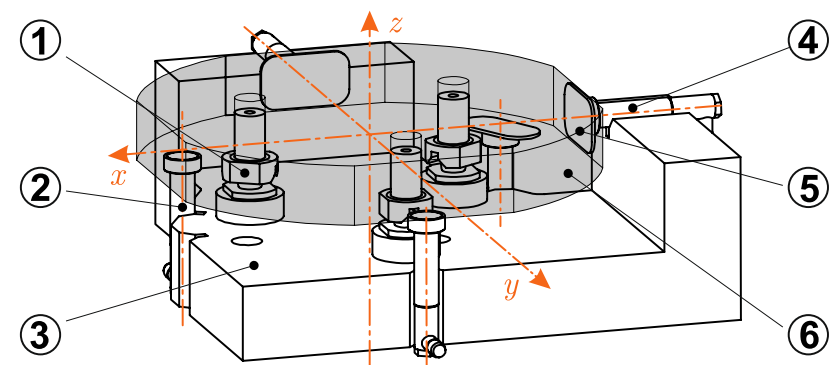

Fig. 8 Metrological components of the measurement setup without the sensor clamping elements: (1) interchangeable inserts; (2) $z$-sensor in V-groove; (3) static half of the metrological frame; (4) $x$-, $y$-sensor in V-groove; (5) capacitor measuring plate; (6) moving half of the metrological frame

designed to fix the five capacitive sensors and is made monolithic to avoid uncertainties due to contact points. For the same reason, the kinematic coupling is also integrated directly into the metrological frame, which means that forces must be taken by the frame. Therefore, the metrological frame is made as massive parts of Zerodur ${ }^{\circledR}$, which is characterized by a relatively high Young's modulus of 90.3 GPa in addition to its low thermal expansion [21]. Based on the one-axis test in chapter $6.1, \mathrm{~V}$-grooves are added into the static half of the metrological frame for the sensor clamping and the sensors are adjusted into the working range using gauge blocks. The kinematic coupling is integrated into the metrological frame as exchangeable elements to vary the materials, shapes, and sizes of the coupling elements. These cylindrical exchangeable coupling elements are mounted to the metrological frame via very well-defined holes and are secured against rotation by spring forces. These geometries are chosen because the force application to the frame is more crucial than the position definition of the exchangeable coupling elements.

\subsection{Lift-off Device}

Since the sensors have a working distance of only $50 \mu \mathrm{m}$ , the high demands made on the guidance are achieved through a double diaphragm spring guide (see Fig. 9) characterized by very small guidance deviations and a high rigidity against tilting. To prevent the stiffness of the guidance system from becoming too high and the actuator from being unnecessarily loaded, the diaphragm springs are axially slotted.

The connection to the moving coupling half is realized by three ball-plane contacts. To prevent possible tilting during lift-off, the three ball-plane contacts must be adjusted in two rotatory directions using two adjustment tables placed at an angle of $90^{\circ}$ above each other. The lift-off device can be engaged by a bayonet-type mount 


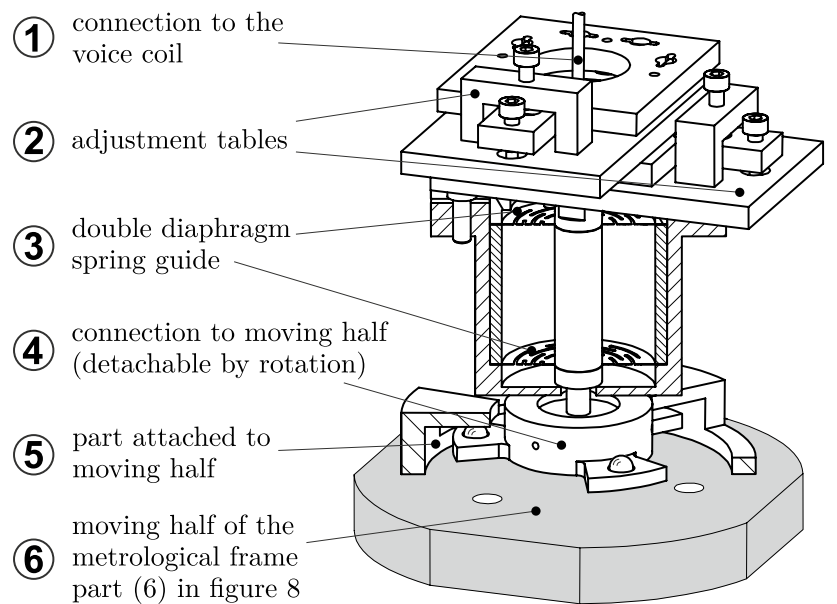

Fig. 9 Lift-off device based on a diaphragm spring guide for an automated measurement in vacuum

allowing quick and easy mounting. A voice coil is used as the actuator and the connection to the diaphragm spring guide is made by a flexible element to avoid overdetermination. Besides, the large distance between the actuator and sensors keeps the influence of the heating of the actuator on the measurement uncertainty small.

\section{Conclusions and Outlook}

In the state of the art, similar measurement setups are already presented, however, either the resolution of the measuring device is too low or the measurement was not performed in at least five DOF. The lift-off and coupling of the two coupling halves were realized manually in many previous works, which results in additional uncertainty contributions that are difficult to consider. The performance of the measurement setup for the intended application can only be proven by a systematic measurement uncertainty analysis. This has not yet been carried out for any of the measurement setups presented in other works. Therefore, a measurement setup has been developed for the determination of the reproducibility of highest precision kinematic couplings with an uncertainty of about $10 \mathrm{~nm}$ per coordinate axis and about $11 \mathrm{nrad}$ per rotation under the given conditions $(k=1)$. This is achieved by a spartan kinematic design, largely reduced number of components, using high-precision capacitive sensors and low thermal expansion material for all metrological components. Since the above-mentioned uncertainty is still a conservative estimate, the expected measurement uncertainty will be smaller. Thereby, the reproducibility of kinematic couplings can be investigated with an uncertainty that has not been achieved before. Further, the use of the measurements in a vacuum environment enables minimization of disturbing influences on the reproducibility.
The measurement setup is currently being mounted and set into operation. Subsequently, it will be used to investigate different variants of kinematic couplings in a vacuum using the exchangeable coupling elements. A particular focus is on the change in friction conditions caused by different materials and the vacuum environment. Furthermore, the influence on the reproducibility by the application of lubricants or high-frequency vibrations will be investigated too.

Acknowledgements The authors gratefully acknowledge the support by the Deutsche Forschungsgemeinschaft (DFG) in the framework of Research Training Group "Tip- and laser-based 3D-Nanofabrication inextended macroscopic working areas" (GRK 2182) at the Technische Universiät Ilmenau, Germany.

Availability of data and materials The research data are available upon request to the authors.

\section{Declarations}

Conflict of interest The authors declare that they have no conflicts of interest.

Open Access This article is licensed under a Creative Commons Attribution 4.0 International License, which permits use, sharing, adaptation, distribution and reproduction in any medium or format, as long as you give appropriate credit to the original author(s) and the source, provide a link to the Creative Commons licence, and indicate if changes were made. The images or other third party material in this article are included in the article's Creative Commons licence, unless indicated otherwise in a credit line to the material. If material is not included in the article's Creative Commons licence and your intended use is not permitted by statutory regulation or exceeds the permitted use, you will need to obtain permission directly from the copyright holder. To view a copy of this licence, visit http://creativecommons.org/licenses/by/4.0/.

\section{References}

1. Jäger G, Manske E, Hausotte T, Müller A, Balzer F (2016) Nanopositioning and nanomeasuring machine NPMM-200-a new powerful tool for large-range micro- and nanotechnology. Surf Topogr Metrol Prop. https://doi.org/10.1088/2051-672X/4/3/ 034004

2. Ortlepp I, Kühnel M, Hofmann M, Weidenfeller L, Kirchner J, Supreeti S, Mastylo R, Holz M, Michels T, Roland Füßl, Rangelow IW, Fröhlich T, Dontsov Denis, Schäffel C, Manske E (2020) Tip- and laser-based nanofabrication up to $100 \mathrm{~mm}$ with sub-nanometre precision. In: Proc Vol 11324, p. 113240A. Int Soc for Opt and Photonics. https://doi.org/10.1117/12.2551044

3. Füß1 R, Grünwald R, Schmidt I (2006) Analysis of the measurement uncertainties of nanopositioning and nanomeasuring machines (NPM-machines) by means of a new vectorial model approach. Tech Mess en 73(9):465-471

4. Niven WD, Maxwell JC (1890) The Scientific Papers of James Clerk Maxwell:, vol 2. Cambridge University Press, Cambridge, United Kingdom, Camb Libr Collect - Phys Sci. https://doi.org/ 10.1017/CBO9780511710377

5. Kelvin WT, Tait PG (1879) Treatise on natural philosophy, reprinted, 1912th edn. Cambridge University Press, Cambridge, United Kingdom, Camb Lib Collect—Math 
6. Hexagon Manufacturing Intelligence: Leitz PMM-C Line (2017). http://www.hexagonmi.com/de-DE/products/coordinate-measu ring-machines/bridge-cmms/leitz-infinity

7. Slocum A (1988) Kinematic couplings for precision fixturingpart I: formulation of design parameters. Precis Eng 10(2):85-91. https://doi.org/10.1016/0141-6359(88)90005-0

8. Slocum AH, Donmez A (1988) Kinematic couplings for precision fixturing — part 2: experimental determination of repeatability and stiffness. Precis Eng 10(3):115-122. https://doi.org/10.1016/01416359(88)90029-3

9. Grimske S, Kong N, Röhling B, Wulfsberg JP (2012) Repeatable positioning accuracy of mechanical interfaces for micro manufacturing. Proceedings of 12th EUSPEN Conference, pp 209-212

10. Horvath NW, Davies MA, Patterson SR (2019) Kinematic mirror mount design for ultra-precision manufacturing, metrology, and system level integration for high performance visible spectrum imaging systems. Precis Eng 60:535-543. https://doi.org/10. 1016/j.precisioneng.2019.09.011

11. Ziegert J, Tymianski V (2007) Air bearing kinematic couplings. Precis Eng 31(2):73-82. https://doi.org/10.1016/j.precisioneng. 2006.02.006

12. Saha SK, Uphaus TM, Cuadra JA, Divin C, Ladner IS, Enstrom KG, Panas RM (2018) Kinematic fixtures to enable multi-material printing and rapid non-destructive inspection during two-photon lithography. Precis Eng. https://doi.org/10.1016/j.precisioneng. 2018.05.009

13. Ng TW (2009) Evaluation of kinematic coupling stability via centroid tracking of a lighted pinhole. Precis Eng 33(2):214-216. https://doi.org/10.1016/j.precisioneng.2008.05.002
14. Leach RK (2010) Fundamental principles of engineering nanometrology, 1st edn. Micro and nano technologies. Elsevier, Amsterdam

15. Schuth M, Buerakov W (2017) Handbuch optische Messtechnik: Praktische Anwendungen für Entwicklung, Versuch Fertigung und Qualitätssicherung. Hanser, München

16. Mastylo R, Jäger G, Linß G, Bahrs U (2012) Optische und taktile Nanosensoren auf der Grundlage des Fokusverfahrens für die Anwendung in Nanopositionier- und Nanomessmaschinen: @ Ilmenau, Techn. Univ., Diss., 2012. Univ.-Bibliothek, Ilmenau

17. Ullmann V, Oertel E, Manske E (2018) High-precision angle sensor based on a Köster's prism with absolute zero-point. Measurement Science and Technology 29(6). https://doi.org/10.1088/ 1361-6501/aab252

18. Kühnel M, Fern F, Fröhlich T (2018) Novel monolithic pendulum tiltmeter with nanorad resolution. Tech Messen 85(4):244-251. https://doi.org/10.1515/teme-2017-0097

19. Fern F, Füßl R, Eichfelder G, Manske E, Kuehnel M (2020) Coordinate transformation and its uncertainty under consideration of a non orthogonal coordinate base. Meas Sci Tech. https://doi.org/ 10.1088/1361-6501/aba3f5

20. Physik Instrumente: D-510 piseca capacitive sensors (2020). https://www.physikinstrumente.de/de/produkte/sensoren-kompo nenten-zubehoer/e-852-piseca-sensorauswertung-500500/\#varia nts

21. Schott: Zerodur ${ }^{\circledR}:$ Zero expansion glass ceramic (2011). https:// www.schott.com/d/advanced_optics/f7ae3c11-0226-4808-90c759d6c8816daf/1.3/schott_zerodur_katalog_july_2011_en.pdf 